\title{
Acessibilidade à Mamografia para rastreamento e prevenção do Câncer de Mama em Mulheres Jovens e Idosas: Um Relato de Experiência
}

\author{
Rayanne de Lima Capistrano ${ }^{1}$; Susanne Pinheiro Costa e Silva ${ }^{2}$
}

\begin{abstract}
Resumo: Objetivo: Relatar a experiência da acessibilidade à mamografia no município de Serrinha-Ba, contextualizando como se dá o rastreamento e prevenção do câncer de mama em caminhões itinerantes. Método: Trata-se de um estudo qualitativo e descritivo do tipo relato de experiência, no período de outubro a novembro de 2017. Resultados e Discussão: A gestão do atendimento distribuiu a demanda a partir dos territórios da Estratégia Saúde da Família e por bairros. Foram realizados aproximadamente 2100 exames de mamografia neste período. Dentre estes, em cerca de 20 exames foram detectadas alterações que necessitavam de encaminhamento para especialistas. A entrega de resultados foi realizada via Secretaria de Saúde, em parceria com os Agentes Comunitário de Saúde. Conclusão: A ação contribuiu para facilitar o acesso das mulheres ao exame mamográfico, auxiliando largamente na aproximação do serviço de saúde com a usuária para a prevenção do câncer de mama e detcção precoce. O estudo mostra a importância de ações como esta, na oferta de serviços especializados, beneficiando a comunidade, instituições envolvidas e profissionais do setor saúde.
\end{abstract}

Palavras-Chave: Mamografia; Câncer de Mama; Exame Diagnóstico; Relato de Experiência.

\section{Mammography Accessibility for Screening and Prevention of Breast Cancer in Young and Older Women: An Experience Report}

\begin{abstract}
Objective: To report the experience of the accessibility to mammography in the city of Serrinha-Ba, contextualizing how the tracking and prevention of breast cancer occurs in itinerant trucks. Method: This is a qualitative and descriptive study of the type of experience report, from October to November, 2017. Results And Discussion: The management in the care was distributed by territories of the Family Health Strategy and by neighborhoods. Approximately 2100 mammography examinations were performed in this period, of which about 20 examinations were detected. The delivery of results was done through the Health Department, in partnership with the Community Health Agents. Conclusion: the Action contributed to facilitate the access of women to the mammographic examination, greatly assisting the approach of the health service with the user in the early detection and prevention of breast cancer. The study shows the importance of actions such as this, in the provision of specialized services, benefiting the community, institutions involved and professionals in the health sector.
\end{abstract}

Keywords: Mammography; Breast Cancer; Diagnostic Examination; Experience Report.

\footnotetext{
${ }^{1}$ Enfermeira. Pós Graduada em Enfermagem em Urgência e Emergência. Pós-Graduanda em Gestão em Saúde - UNIVASF (PE). Endereço: Rua Henrique de Menezes, 394, Bairro Centro, Serrinha/BA. Brasil. CEP 48700-000. Telefone: (75)991895496. E mail: raylcapistrano@hotmail.com

${ }^{2}$ Doutora em Psicologia. Professora do Mestrado Profissional em Gerontologia. Universidade Federal da Paraíba. João Pessoa, Paraíba, Brasil. ORCID iD: https://orcid.org/0000-0002-9864-3279. E-mail: susanne.pc@gmail.com.
} 


\section{Introdução}

O câncer de mama é uma patologia consequente da multiplicação de células anormais, formando um tumor com potencial para invadir outros órgãos. Há vários tipos de câncer de mama, sendo que alguns se desenvolvem mais rapidamente que outros. É um considerável problema de Saúde Pública, especialmente por ser o tipo mais comum entre as mulheres no mundo e no Brasil, depois do de pele não melanoma. No Brasil, foram estimados 57.960 casos novos em 2016, que são cerca de 30\% dos cânceres femininos e representam o tipo de câncer mais incidente e a primeira causa de morte por câncer entre as mulheres brasileiras de quase todas as grandes regiões do país. Ainda assim, a alta incidência está diretamente relacionada à falta de informação (BRASIL, 2016; BRASIL, 2018; BRASIL, 2017).

De acordo com o Instituto Nacional de Câncer - INCA, os exames para detecção precoce do câncer de mama mais eficazes são o exame clínico e a mamografia. $\mathrm{O}$ autoexame das mamas detecta a doença geralmente em estádio avançado. O exame clínico faz parte do atendimento integral à mulher, devendo ser inserido no exame físico e ginecológico de todas as mulheres, independente da faixa etária, servindo de subsídio para exames complementares (SILVA, 2011).

Já a mamografia é um exame radiológico para avaliação das mamas que pode identificar lesões benignas e cânceres, os quais geralmente se apresentam como nódulos ou calcificações, sendo este compreendido como parte do atendimento integral à saúde da mulher, capaz de identificar tumores menores que $01(\mathrm{um}) \mathrm{cm}$ e imperceptíveis ao toque, fase da doença que as chances de cura chegam a 95\%. A necessidade de um exame de mamografia depende de alguns fatores como a idade, sintomas e histórico médico da cliente (SILVA, 2011; FÉLIX, 2017; JAGUARÃO, 2013).

Entre os estudiosos do assunto, há ampla concordância de que o rastreamento mamográfico reduz a mortalidade pelo câncer de mama em mulheres assintomáticas, incluindo ainda outros benefícios através da detecção precoce, como o aumento das opções terapêuticas, da probabilidade de sucesso do tratamento e da sobrevida. Muitas vezes, a não adesão ao exame pode ocorrer pelo elevado custo, além das extensas filas de agendamento para realizá-lo em algumas localidades através do Sistema Único de Saúde - SUS (JAGUARÃO, 2013). 
As recomendações para detecção precoce do câncer de mama no Brasil, atualizadas em 2015, propõem o diagnóstico precoce. O INCA recomenda a mamografia de rastreamento para as mulheres entre 50 e 69 anos, sendo apenas um exame a cada dois anos. O diagnóstico precoce é formado pelo tripé: população alerta para os sinais e sintomas suspeitos; profissionais de saúde capacitados para avaliar os casos suspeitos; e sistemas e serviços de saúde preparados para garantir a confirmação diagnóstica oportuna e com qualidade. Já as mulheres que têm casos de câncer de mama na família terão de realizar esse exame uma vez por ano a partir dos 35 anos de idade (FÉLIX, 2017; TOMAZELLI, 2016; BRASIL, 2015).

Para o Brasil, estimam-se 59.700 casos novos de câncer de mama, para cada ano do biênio 2018-2019, com um risco estimado de 56,33 casos a cada 100 mil mulheres. Ações de controle do câncer de mama no Brasil vêm sendo progressivamente incorporadas às políticas públicas de saúde desde o final da década de 1980. No intuito de modificar esse cenário, o controle do câncer de mama tem sido uma das prioridades na agenda da Política Nacional de Saúde do Brasil. Assim, o Ministério da Saúde recomenda a identificação da doença em estágios iniciais por intermédio das estratégias de detecção precoce, pautadas nas ações de rastreamento e diagnóstico precoce (TOMAZELLI, 2016; INCA, 2017).

Concomitante às Diretrizes para a detecção precoce do câncer de mama no Brasil, a União instituiu, através da Portaria $n^{\circ}$ 2.304/2012, no intuito de ampliar a qualidade da assistência e o número dos serviços oncológicos no País, o Programa de Mamografia Móvel no âmbito do Sistema Único de Saúde (SUS), mais conhecido como Programa de Mamografia Móvel. Esta iniciativa consiste na liberação de Unidades Oncológicas volantes, que percorrem locais estratégicos dos municípios, para a realização das mamografias. Não há como negar que este programa facilita o acesso das usuárias que necessitam do diagnóstico (BRASIL, 2012).

No ano de 2017, um dos municípios escolhidos fora a cidade de Serrinha, localizada na Bahia, a $173 \mathrm{~km}$ da capital Salvador, motivado pelo movimento popularmente conhecido como "Outubro Rosa". Este é renomado internacionalmente e comemorado em todo o mundo. Iniciou nos Estados Unidos, na década de 1990, para estimular a participação da população no controle do câncer de mama, tendo o objetivo de compartilhar informações sobre o câncer de mama e promover a conscientização acerca da importância da detecção precoce da doença (BRASIL, 2015). 
Isto posto, este trabalho tem como objetivo relatar a experiência da acessibilidade à mamografia no referido municipio, contextualizando como se dá o rastreamento e prevenção do câncer de mama em caminhões itinerantes.

\section{Método}

Trata-se de um estudo qualitativo e descritivo do tipo relato de experiência, culminado a partir da prática como preceptora de estágio de disciplina do curso técnico de Enfermagem no Hospital Municipal de Serrinha, Bahia, no período de outubro a novembro de 2017.

O Hospital Municipal de Serrinha-Ba realiza semanalmente 50 (cinquenta) exames mamográficos, embora no período compreendido entre os meses de outubro e novembro, durante a campanha do "Outubro Rosa". Este serviço ocorria nos caminhões itinerantes da Secretaria de Estado da Saúde da Bahia - SESAB, através do qual se realiza mutirão de mamografias, emergindo inclusive a necessidade de voluntários para auxiliar no mesmo.

A metodologia abordada baseou-se no relato de ações voluntárias no caminhão itinerante da SESAB, cujo propósito foi contribuir com o processo de realização de mamografias como exame preventivo e diagnóstico para o câncer de mama. Durante todo o período de voluntariado, foram observadas as idas e vindas de mulheres em busca do exame, que demandam uma logística diferendiada para o atendimento às usuárias.

O atendimento no caminhão foi expecífico para mulheres entre 50-69 anos, grupo em que o Ministério da Saúde indica investigação a cada dois anos. Os atendimentos foram iniciados e programados para finalizar em determinada data, que necessitou ser prorrogada em mais uma semana pela alta demanada. O hórario de funcionamento também precisou ser aumentado pelo mesmo motivo, estendendo-se em determinados dias até às $22 \mathrm{~h}$, quando deveria ter sido encerrado às $18 \mathrm{~h}$.

\section{Resultados e Discussão}

Iniciando as atividades, na primeira semana o movimento contou com a presença de 2(dois) caminhões que possuíam uma equipe com 2(dois) técnicos em radiologia em cada carro, realizando 240 exames de mamografia. Com o passar dos dias, a demanda foi sendo reduzida gradativamente, ficando no município apenas 1(um) caminhão móvel, passando então a ocorrer 140 exames diários de mamografia. O expediente era de segunda-feira a sábado, porém a 
população não aderiu aos dias de sábado e as atividades funcionaram normalmente de segunda à sexta-feira, tendo intervalo aos fins de semana.

A gestão no atendimento foi distribuído por territórios da Estratégia Saúde da Família (ESF) e por bairros. As mulheres que pertenciam às áreas descorbertas se cadastravam como residentes na macroregião mais próxima ao bairro ou da comunidade na qual viviam, no intuito de realizar o rastreamento do câncer de mama no número máximo de mulheres que fosse possível.

Mulheres que residiam na zona rual eram atendidas no turno matutino; mulheres que residiam em área urbana foram atendidas no período vespertino. Essa organização teve o apoio dos Agentes Comunitários de Saúde (ACS), que facilitaram a identificação das macroregiões e das mulheres. No último dia a demanada foi espontânea, justamente para aquelas mulheres que não foram assistidas por quaisquer motivos durante o período de agendamento para a sua área.Para realizar o exame fazia-se necessário possuir em mãos a foto cópia da Identidade, o comprovante de residência e cartão SUS. Para aquelas mulheres que ainda não o possuíam, foi disponibilizado um ponto de apoio para a realização do mesmo, facilitando o atendimento, e evitando uma possível disistência por falta de documentação. Assim sendo, o atendimento às mulheres possuía um fluxograma dinâmico (Figura 1). 
Figura 1 - Fluxograma de Atendimento da Campanha Outubro Rosa em Serrinha-BA

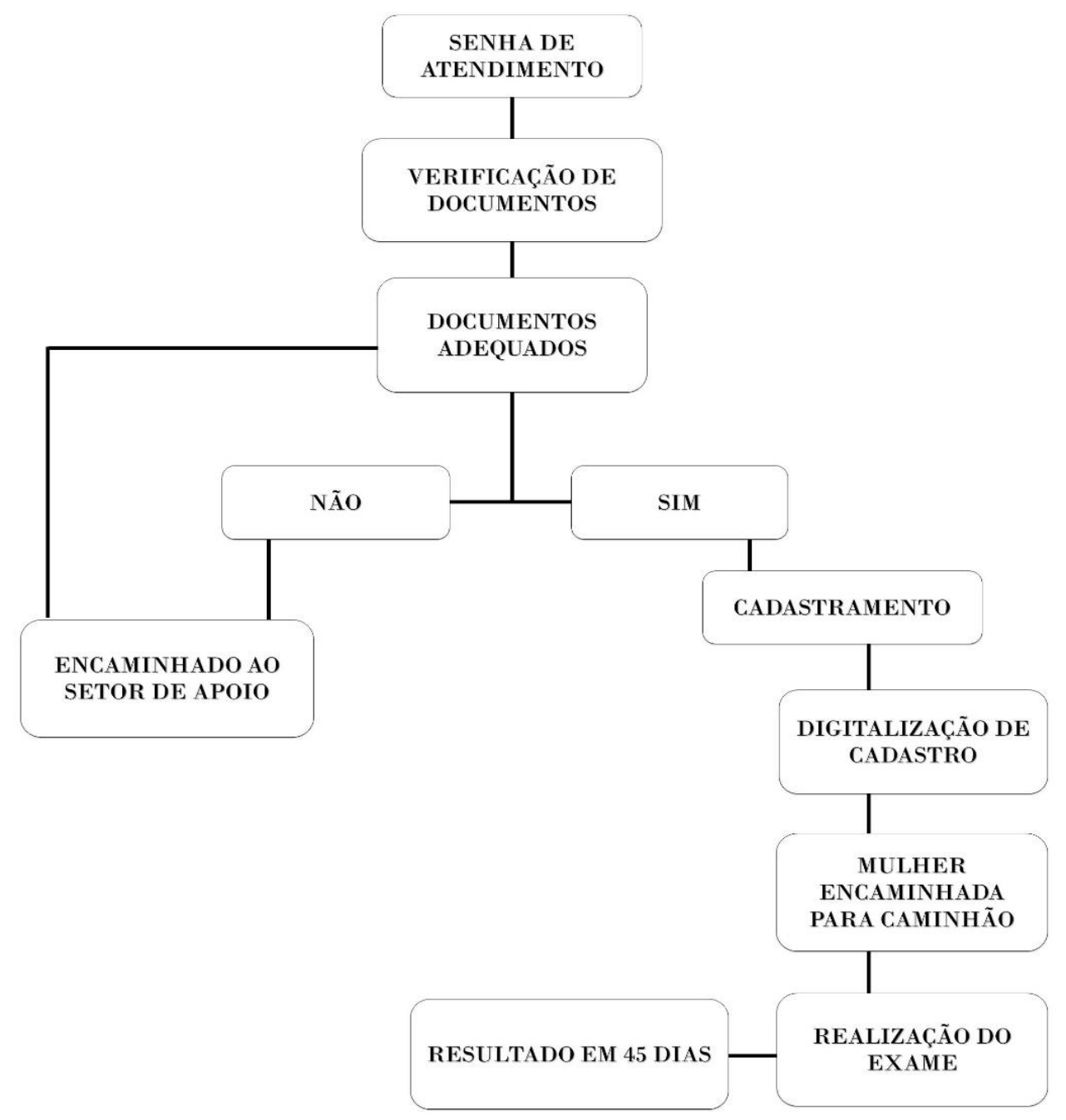

O relatório médico era confeccionado por meio da telemedicina, onde o exame é avaliado e entregue de forma digital, dando apoio para a medicina tradicional, funcionando 24 horas por dia. Os laudos são gerados e impressos por uma empresa terceirizada; os resultados contêm laudos com assinatura digital e películas mono-emulsionadas, tendo previsão de entrega em até 45 dias da realização do exame. Como logística para entrega destes resultados, às mulheres se dirigem à Secretaria Municipal de Saúde do município e lá resgatam o seu exame, desde que estejam portando documento com foto.

Foram realizados aproximadamente 2100 exames de mamografia neste período. Dentre estes, em cerca de 20 exames foram detectadas alterações. Nestes casos, a clínica responsável 
entrou em contato com a Secretaria de Saúde do Município que, por sua vez, realizou a busca ativa das mulheres para realizarem o procedimento de punção mamária, encaminhando-as para oncologistas a fim de concluir o diagnóstico.

A entrega de resultados foi realizada via Secretaria de Saúde, em parceria com os ACS. Muitas das mulheres foram pegar os seus resultados na própria secretaria; outras receberam atraves do ACS. Por sua vez, diversas mulheres não foram retirar seus exames. Fazendo um comparativo com anos anteriores, no ano de 2017 houve maior adesão na realização da mamografia e a entrega de resulltados foi mais eficiente.

Após receber os resultados sem alterações, as mulheres eram orientadas para apresentar o exame ao ginecologista. Em diálogo com algumas usuárias, houve o relato de que este programa facilitou o acesso das mulheres ao exame de mamografia, mesmo que o municipio forneça o exame durante o ano inteiro, ja que a demanda é alta para a disponibilidade do número vagas para as mulheres que necessitam realizá-lo.

No tempo de espera para a realização do exame, as mulheres conversavam entre elas e recebiam orientações para a realização da mamografia. Nos diálogos entre si e com a equipe de trabalho, as mesmas confessavam medo, especialmente pelo fato de outras discorrerem sobre dor na execução do exame, principalmente quando o realizavam pela primeira vez. Outras elogiaram o fluxo do atendimento pela sua agilidade, orientações pertinentes e o atendimento humanizado.

\section{Conclusão}

O projeto das mamografias móveis contribuiu para facilitar o acesso das mulheres ao exame mamográfico, auxiliando alargamente a aproximação do serviço de saúde com a usuária na detcção precoce e prevenção do câncer de mama. Dentro dessa perspectiva, destaca-se a necessidade do retorno do projeto nos municípios anualmente, para que haja continuidade na oferta, não bastando investir em equipamentos apenas, fazendo-se necessário ampliar a disseminação de informações sobre a importância da mamografia. Foi notória a adesão do exame por parte das municipes da zona urbana, muito mais do que as residentes em zona rural. Os gestores municipais de saúde enfatizaram a qual grande importância nesta parceria com o Estado, viabilizando e facilitando a ação dos cominhões intinerantes. Desta forma, o estudo 
mostra a importância de ações como esta, na oferta de serviços especializados, beneficiando a comunidade, instituições envolvidas e profissionais do setor saúde.

Teve-se como limitação da pesquisa o tempo para coleta das informações, porém o levantamento de dados foi válido, ja que permitiu a explanação da acessibilidade ao exame de mamografia em períodos cruciais como no mês dedicado ao rastreamento do Câncer de Mama através das ações do Outubro Rosa.

\section{Referências}

BRASIL. Ministério da Saúde. Instituto Nacional de Câncer José Alencar Gomes da Silva. O movimento Outubro Rosa. Rio de Janeiro, 2015. Disponível em: <http://www.inca.gov.br/wcm/outubro-rosa/2015/movimento-outubro-rosa.asp> Acessado em 08 de Agosto de 2018.

BRASIL. Ministério da Saúde. Instituto Nacional de Câncer José Alencar Gomes da Silva. Controle do Câncer de mama: Conceito e magnitude. Rio de Janeiro, 2017. Disponível em:<http://www2.inca.gov.br/wps/wcm/connect/acoes_programas/site/home/nobrasil/progra ma_controle_cancer_mama/conceito_magnitude>. Acessado em 10 de Junho de 2018.

BRASIL. Ministério da Saúde. Instituto Nacional de Câncer José Alencar Gomes da Silva. Estimativa 2018: incidência de câncer no Brasil. Coordenação de Prevenção e Vigilância. Rio de Janeiro: INCA, 2017.2 Disponível em: <http://www1.inca.gov.br/inca/Arquivos/estimativa-2018.pdf >. Acessado em 11 de Junho de 2018.

BRASIL. Ministério da Saúde. Instituto Nacional de Câncer José Alencar Gomes da Silva. Câncer de Mama. Disponível em: <http://www2.inca.gov.br/wps/wcm/connect/tiposdecancer/site/home/mama/cancer_mama+> Acessado em 11 de Junho de 2018.

BRASIL. Ministério da Saúde. Instituto Nacional de Câncer José Alencar Gomes da Silva. Estimativa 2016: incidência de câncer no Brasil. Rio de Janeiro, 2015. Disponível em: $<$ http://santacasadermatoazulay.com.br/wp-content/uploads/2017/06/estimativa-2016v11.pdf $>$. Acessado em 11 de Junho de 2018.

BRASIL. Ministério da Saúde. Instituto Nacional de Câncer José Alencar Gomes da Silva. Tipos de Cancer: Mama. Disponível em: <http://www2.inca.gov.br/wps/wcm/c onnect/tiposdecancer/site/home/mama/cancer_mama+> . Acessado em 15 de maio de 2018.

BRASIL. Ministério da Saúde; Instituto Nacional de Câncer José Alencar Gomes da Silva; Câncer de mama: é preciso falar disso. 4. ed. - Rio de Janeiro: Inca, 2016. 
BRASIL. Ministério da Saúde; Portaria no 2.304, de 4 de outubro de 2012. Disponível em < ht tp://bvsms.saude.gov.br/bvs/saudelegis/gm/2012/prt2304_04_10_2012.html> . Acessado em 15 de maio de 2018.

FÉLIX, J.; et.al. Mamografia: Aspectos Gerais. Revista Científica Multidisciplinar Núcleo do Conhecimento. Ano 2, Vol. 13. pp 447-454 Janeiro de 2017. ISSN:2448-0959.

JAGUARÃO. Câmara de Vereadores de Jaguarão. Câncer De Mama. Disponível em: $<$ https://www.fundacaoulysses.org.br/wp-content/uploads/img-pdf/1397141709-1389293704miolo-cancer-de-mama.pdf >. Acessado em 30 de maio de 2018.

SILVA, P. A.; RIUL, S. da S. Câncer de mama: fatores de risco e detecção precoce. Rev. bras. enferm. [online]. 2011, vol.64, n.6, pp.1016-1021. ISSN 0034-7167. Disponível em: <http://dx.doi.org/10.1590/S0034-71672011000600005> Acessado em 30 de maio de 2018.

TOMAZELLI, J. G. et al. Avaliação das ações de detecção precoce do câncer de mama no Brasil por meio de indicadores de processo: estudo descritivo com dados do Sismama, 2010-2011.Epidemiol. Serv. Saúde [online]. 2017, vol.26, n.1, pp.61-70. Epub Nov 28, 2016. ISSN 1679-4974. Disponível em: <http://dx.doi.org/10.5123/s1679-49742017000100007>. Acessado em 10 de Junho de 2018.

\section{Como citar este artigo (Formato ABNT):}

CAPISTRANO, Rayanne de Lima; SILVA, Susanne Pinheiro Costa e. Acessibilidade à Mamografia para rastreamento e prevenção do Câncer de Mama em Mulheres Jovens e Idosas: Um Relato de Experiência. Id on Line Rev.Mult. Psic., 2018, vol.12, n.42, p. 93-101. ISSN: 1981-1179.

Recebido: 20/08/2018.

Aceito: 23/08/2018 